\title{
Le danseur et le musicien, une connivence nécessaire
}

\section{Anca Giurchescu}

Traducteur : Ramèche Goharian

\section{OpenEdition \\ Journals}

\section{Édition électronique}

URL : http://journals.openedition.org/ethnomusicologie/99

ISSN : 2235-7688

Éditeur

ADEM - Ateliers d'ethnomusicologie

Édition imprimée

Date de publication : 1 décembre 2001

Pagination : 79-94

ISBN : 2-8257-07-61-9

ISSN : 1662-372X

Référence électronique

Anca Giurchescu, «Le danseur et le musicien, une connivence nécessaire », Cahiers d'ethnomusicologie [En ligne], 14 | 2001, mis en ligne le 10 janvier 2012, consulté le 10 décembre 2020. URL : http:// journals.openedition.org/ethnomusicologie/99

Ce document a été généré automatiquement le 10 décembre 2020.

Tous droits réservés 


\title{
Le danseur et le musicien, une connivence nécessaire
}

\author{
Anca Giurchescu \\ Traduction : Ramèche Goharian
}

\section{Un débat culturel}

1 On croit d'habitude que chaque forme d'art est unique et que les sentiments et les idées exprimés par une forme ne peuvent être remplacés par aucune autre. Cependant, d'un point de vue cognitif plus général, on peut concevoir qu'il existe des règles d'organisation et des capacités expressives communes pour deux formes d'art, voire plus. Il arrive qu'à un certain stade de leur histoire et dans des contextes sociaux particuliers, des gens appartenant à une culture donnée établissent un lien entre différentes formes d'art et que ce lien se stabilise et se développe avec le temps. Le débat sur le rapport entre la danse et la musique de danse provient d'un choix culturel déterminé, caractéristique du concept européen de folklore. Les deux formes artistiques se trouvent ici arbitrairement isolées des autres modes d'expression humaine avec lesquelles elles coexistent et établissent des échanges dans un contexte social donné.

2 Envisagée dans une perspective diachronique, la danse apparaît comme un phénomène artistique complexe qui, dans les cultures traditionnelles, se trouve intimement lié à d'autres genres et à d'autres modes d'expression d'origines diverses. On peut imaginer qu'à ses débuts la danse impliqua une combinaison indifférenciée de mouvements corporels, de gestes et d'exclamations syllabiques à forte consonance musicale. Lorsque cet «amalgame » indifférencié donna naissance à la danse, à la musique et à la poésie dans des modalités expressives au contour plus précis, ces formes d'art eurent, toutes ensemble ou certaines d'entre elles, une existence syncrétique. C'est ce que l'on observe encore aujourd'hui en étudiant une culture traditionnelle. Avec le temps, chacun de ces genres artistiques est devenu autonome et autosuffisant ou, en d'autres termes, aucun de ces modes fondamentaux d'expression ne peut contribuer à la définition d'un autre mode. 
Ce qui unifie la danse (mouvement corporel organisé dans l'espace), le texte (émission de syllabes organisées) et la musique (sons organisés dans le temps), c'est le rythme, défini par Platon comme « l'ordre du mouvement » (Ciobanu 1979). C'est donc grâce au rythme que ces trois phénomènes artistiques essentiels peuvent, dans certains cas, exister et établir des échanges dans un syncrétisme qui est profondément enraciné dans l'histoire de la culture. Le terme musikë d'où dérive notre mot musique recouvrait chez les anciens grecs le sens global de chanter, réciter de la poésie, mouvoir le corps, danser et manipuler des instruments (Stockmann 1985 : 17). De même le terme choros qui, de nos jours, désigne seulement la danse, vient de choreia qui signifiait à la fois l'unité de la danse et du chant et le groupe d'artistes qui les interprétaient (Michaelides 1978) ${ }^{1}$. L'utilisation des mots pous en grec et pes en latin qui veulent dire pied illustrent le rôle essentiel que joue le mouvement dans la structure métrique des vers (Sachs 1953 : 38).

3 Avec le temps, l'amalgame indifférencié et fondamental se désintégra et, dans le cas de la tradition culturelle européenne, il ne reste plus que quelques exemples du syncrétisme originel. On peut le retrouver dans certains jeux d'enfants et dans des jeux chantés basés essentiellement sur le mouvement. Cependant les formes artistiques autonomes comme la musique, la danse et la poésie peuvent s'associer à nouveau dans des contextes sociaux traditionnels selon leur affinité initiale (Mîrza $1972: 235)^{2}$.

4 La pertinence de la relation danse-musique n'apparaît pas de la même façon dans toutes les cultures et dans tous les contextes sociaux-culturels. Par essence, cette relation est culturellement déterminée : il existe de nombreux exemples de danse sans musique et de musique de danse interprétée sans la danse dans les contextes artistiques traditionnels ou classiques. Si nous limitons nos exemples à la tradition culturelle européenne où, comme nous l'avons déjà indiqué, la relation entre la musique et la danse joue un rôle de première importance, l'absence de musique d'accompagnement pour la danse prend un sens très particulier. D'un point de vue historique, par exemple, l'absence totale de chant et d'instruments de musique pour accompagner la danse «silencieuse » d'un couple ou d'une chaîne de danseurs mixtes au Monténégro et en Macédoine est interprétée comme le symbole de la domination turque au XVI ${ }^{\mathrm{e}}$ siècle, lorsque les autochtones n'avaient pas le droit de danser (Kurath $1986: 311)^{3}$. 
Fig. 1 : Filles tsiganes (Băieşi) du village de Szedres (Hongrie) accompagnant des chants dansés, avec des pseudo-instruments rythmiques (cuillères, boîte de fer blanc) et des battements de mains

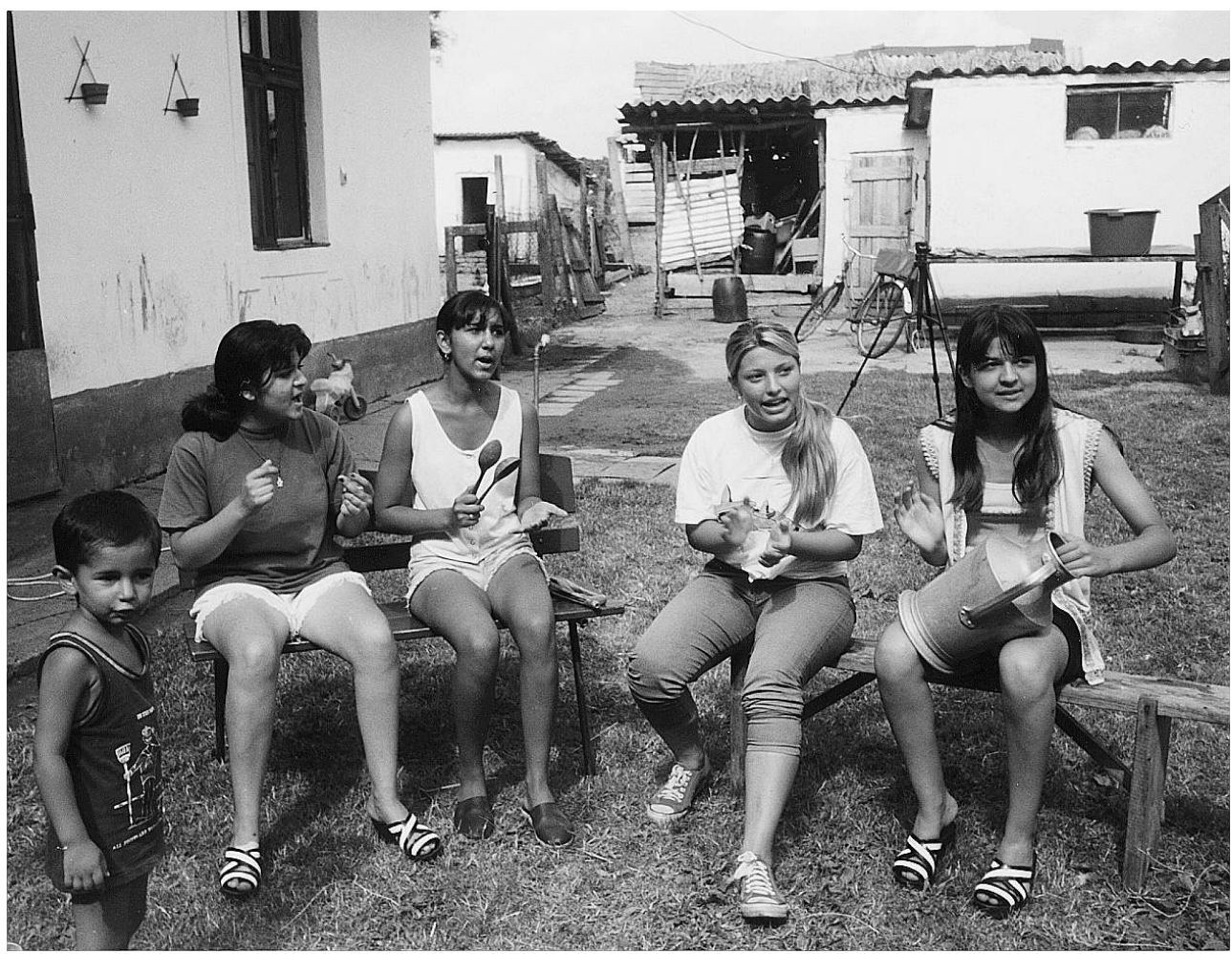

Photo : Anca Giurchescu, juillet 1999

5 Si nous situons notre exposé sur le plan du folklore, nous devons essayer de trouver un concept global expliquant l'unité fondamentale qui caractérise l'existence syncrétique d'activités telles que la poésie, la musique et la danse. Ces activités, qui peuvent fonctionner de façon autonome dans d'autres contextes socioculturels, agissent les unes sur les autres au niveau du folklore comme parties intégrantes d'un " objet sémiotique " unitaire, censé produire une signification globale conforme au contexte social donné (Greimas 1971: 65). Des événements tels que les danses, les mariages ou les funérailles, les cérémonies saisonnières ou les rituels de guérison, de protection ou d'invocation des forces bénéfiques, etc., peuvent être considérés comme des textes culturels multimédias caractérisés par l'interaction de plusieurs modalités expressives et de codes suivant des hiérarchies spécifiques tels que : la musique + la danse + les gestes + la poésie + les costumes + les masques + les accessoires ou les objets + les règles de comportement social et rituel, etc. Envisagées sous cet angle, la danse et la musique sont les principales composantes d'une danse villageoise, soutenues par la poésie (textes chantés ou cris versifiés), les codes d'interaction sociale et les facteurs spatio-temporels. A l'inverse, dans certains contextes rituels, la danse ne fait que soutenir les autres composants. Par exemple, pendant les mascarades de Nouvel An et de Carnaval, la danse sert essentiellement à la circulation des masques; dans le mariage roumain, la danse hora de la mariée montre le rapport de parenté qui relie les participants et sert de support à la distribution de cadeaux qui fait partie du rituel d'intégration; dans la paparuda, rituel d'invocation de la pluie, la danse soutient le texte chanté par une jeune fille vêtue de feuilles vertes. 
6 L'importance des moyens expressifs varie d'une culture à l'autre, démontrant clairement que l'art est moins « universel » qu'on le prétend. Par exemple, dans les danses de Tonga, « les mots - c'est-à-dire le texte - constituent la partie essentielle et la plus importante de tout le spectacle. Comme dans la plupart des musiques traditionnelles polynésiennes la mélodie n'est que secondaire. Les mouvements de la danse servent d'accompagnement visuel à la poésie dans la majorité des danses tongiennes. Contrairement à l'idée que les Occidentaux se font communément de Tonga, ce n'est pas la musique qui accompagne la danse mais plutôt la danse qui accompagne la poésie, et la manière dont les mouvements interprètent la poésie est un aspect important de l'esthétique » (Kaeppler 1993 : 31).

7 Lorsque la danse, la musique et la poésie sont intimement liées, la nature de leur relation ne se dévoile qu'au cours de la danse. Selon les contextes sociaux, il existe plusieurs types de relations tels que : la détermination, l'interdétermination (importance équivalente) et la constellation (rapport lâche). La complexité de ces diverses relations est telle qu'aucune généralisation n'est possible (Giurchescu \& Torp 1995 : 144).

8 En réalité, la question de la primauté de la danse ou de la musique ne me semble pas pertinente, car non seulement elle est changeante selon des critères historiques, géographiques, circonstanciels et structurels, mais parce que la danse et la musique sont, en tant que parties intégrantes d'un événement complexe, en état permanent d'interaction et de modification ${ }^{4}$.

9 L'ethnochoréologue György Martin, qui s'est beaucoup intéressé à la relation de la danse avec la musique de danse, définit les types relationnels suivants :

1. «L'accompagnement rythmique indépendant - la danse n'est pas accompagnée de mélodie, mais de motifs rythmiques sonores ".

2. «L'accompagnement mélodique indépendant - lorsqu'une mélodie chantée ou jouée sur un instrument se joint à la danse sans accompagnement rythmique ».

3. "L'accompagnement complexe qui a le rôle dominant dans la musique de danse hongroise actuelle. Ses éléments mélodiques et rythmiques s'entrelacent en une unité organique» (Martin 1965 : 317 ; Felföldi 1995 : 192).

10 L'accompagnement rythmique indépendant est très complexe et peut être décrit comme un accompagnement rythmique personnel, utilisant le corps du danseur comme un instrument. Par exemple, le claquement des différentes parties de la jambe dans les danses de jeunes gens (feciore ște) de Transylvanie (Roumanie) et dans le Schuhplattler de Bavière, ou le claquement de la poitrine et de la bouche des danses tziganes de Hongrie et de Transylvanie, ainsi que les battements des pieds de la danse rituelle căluş en Roumanie ${ }^{5}$. L'accompagnement rythmique indépendant est parfois réalisé avec des objets sonores (colliers, clochettes, coquillages attachés au corps du danseur), par des éléments sonores du costume ou par des pseudo-instruments tels que des castagnettes, des cuillers, des casseroles etc. (Kealiinohomoku 1965-66). Des effets sonores rythmiques vocaux appelés aussi «musique de bouche» accompagnent habituellement les danses tziganes de Hongrie et des Balkans en même temps que le battement des mains et le claquement des doigts. L'accompagnement est parfois réalisé par les danseurs eux-mêmes mais ce sont les personnes extérieures à la danse qui le produisent la plupart du temps (Martin $1965: 317$, n.4). L'accompagnement rythmique indépendant se fait souvent à l'aide de différents instruments de percussion.

11 L'accompagnement mélodique indépendant est produit par des instruments tels que la flûte, le violon, la clarinette etc., par des chants ou par la combinaison des deux ${ }^{6}$. Dans le 
cas des danses chantées comme par exemple dans celles des femmes roumaines ou hongroises de Transylvanie (Roumanie), les mouvements de la danse sont subordonnés d'abord à la poésie, et ensuite à la mélodie (Giurchescu $1967: 286$ ).

12 L'accompagnement complexe est très répandu dans la danse traditionnelle européenne; il combine deux ou plusieurs des types d'accompagnements déjà cités. Il est certain que dans beaucoup de circonstances les danseurs accordent la priorité à un schéma rythmique qui signale un certain genre de danse muni d'un type correspondant d'accompagnement. L'expression courante des musiciens : «Donne-moi une hora ou une sârba, etc.» pour demander un certain accompagnement instrumental met cette corrélation en évidence. La prédilection d'un type de danse pour un accompagnement rythmique a été étudiée en détail par György Martin (1967 : 322-325).

13 Cependant l'accompagnement rythmique ne correspond pas toujours parfaitement aux schémas rythmiques du type de danse correspondant. Dans la tradition des danses de l'Europe méridionale, les schémas rythmiques des types fondamentaux de danse sont syncopés ou alors utilisent une accentuation à contre-temps ${ }^{7}$.

14 Le phénomène de polyrythmie apparait pendant la performance lorsque la structure rythmique de la danse (et de tous les autres mouvements rythmiques significatifs) se superpose au rythme de la musique (accompagnement et mélodie) voire dans certains cas, à celui des cris versifiés, des paroles ou de simples cris. Il provient d'une interaction consciente entre le danseur et le musicien qui trouve son expression dans l'accentuation à contre-temps et syncopée (cf. l'improvisation). Grâce à sa puissance esthétique, la polyrythmie est un trait qui permet de caractériser un type de danse ou un style régional.

15 Plus subtil encore est le décalage vertical entre le rythme de la musique et celui de la danse. Le phénomène de polymétrie est fréquent dans les danses africaines où les différentes parties du corps bougent et tremblent selon des pulsations différentes. La purtata, danse chantée des jeunes-filles de la Transylvanie centrale, illustre bien le décalage métrique entre le schéma des pas en $2 / 4$ et les chants interprétés par les danseuses en mètre asymétrique 9/16 (Giurchescu $1967: 289$ ).

Le tempo de la musique et de la danse est en principe concordant et constant tout au long de l'interprétation. Une accélération et une alternance de tempos contrastants peuvent toutefois apparaître, laissant leur impact sur la structure de la danse. Ainsi, briser l'unité de la musique de danse en fonction du tempo permet de distinguer une danse traditionnelle «authentique » d'une autre qui est délibérément préconçue et fixée. Par exemple l'utilisation du tempo rubato (flexibilité du tempo et du rythme) qui apparait pendant l'improvisation exprime le « tempo personnel » du danseur. Le tempo peut aussi désigner le type de danse comme la danse «lente » et "rapide» des jeunes gens du répertoire roumain ou les csárdás « lents » et " rapides » du répertoire hongrois, chaque type ayant sa ou ses propres mélodies 8 .

17 Les schémas rythmiques du mouvement représentent les unités structurelles de base (motifs des mouvements rythmiques) qui forment, au fur et à mesure de la composition de la danse, de plus grandes unités situées sur plusieurs niveaux hiérarchiques tels que : les phrases, les sections, les strophes, ceci jusqu'à la forme intégrale de la danse. Le rapport dimensionnel entre les unités structurelles de la danse et de la musique montre différents degrés de coïncidence allant de la concordance dimensionnelle parfaite à la coïncidence totalement non concordante, où les unités de la danse et de la musique évoluent parallèlement en s'ignorant mutuellement, ce qui est plutôt rare. Le degré de 
coïncidence le plus fréquent est la semi-concordance, c'est-à-dire l'absence de concordance à un certain niveau structurel (par exemple les motifs), suivie de concordance à un niveau supérieur (phrases) ou au niveau de la forme intégrale de la danse. Un des exemples les plus souvent mentionnés d'absence de concordance au niveau des motifs est la superposition de schémas de mouvements de structure tripode $(2+2+2)$ avec des chants ou des mélodies instrumentales ayant une organisation bipode $(2+2)$. La structure du mouvement tripode est non seulement la plus répandue, mais elle est aussi la plus ancienne; elle existait sans doute dans la tradition de la danse populaire bien avant d'être définie comme branle simple par Thoinot Arbeau, au XVI siècle (Arbeau 1986; Martin 1973). De nos jours, ce schéma caractérise les danses en chaine de la Bretagne, les ballades en chaîne des Iles Feroe, les danses chantées des femmes d'Europe du Centre et du Sud-Est, ainsi que les danses en chaîne, vocales et instrumentales, des Balkans. D'un point de vue historique, on peut penser que le rapport $3: 2$ entre la danse et la musique est né du passage de la concordance entre le vers hexasyllabique ancien et le schéma de la danse à l'octosyllabe plus récent, comme le montrent les danses chantées des jeunes filles de Hongrie. Pour György Martin, les vers hexasyllabiques et la structure tripodique de la danse sont une réminiscence des proportions médiévales qui alternaient la même mélodie dans des rythmes binaires et ternaires (Martin $1968: 331-333)^{10}$. Le rapport $3: 2$, dit schéma Feroe, doit sa vitalité au fait qu'il possède des points de coïncidence récurrents. Ainsi, n'importe quelle mélodie à structure binaire avec un rythme symétrique ou asymétrique peut s'adapter au schéma tripodique de la danse. C'est le cas des types de danses tels que : la sârba et la danse chantée coconita roumaines, le pravo horo bulgare, le kolo serbe, le lesnoto macédonien, la sta tria grec, le koleso et la karicka slovaques et enfin le ballu tundu et le duru-duru sardes. Il importe de savoir que le schéma tripodique peut également exister dans des rythmes asymétriques $(2+3,3+2+2,2+2+3,2+2+2+3)$ (Torp 1990). 
Fig. 2 : Lindjo, interprétée par un couple du groupe de danse KUD Lijerica du village de Slano (Croatie), accompagnée par un lijerica et le battement rythmique des pieds du musicien



Photo : Anca Giurchescu, juillet 2000

En tant que systèmes indépendants, la danse et la musique développent un large spectre de relations structurelles semi ou non concordantes qui découlent d'une mobilité plus grande des mélodies ne correspondant pas strictement à un certain type de danse. Cette mobilité peut susciter non seulement un décalage métrique mais également le fait que les unités structurelles de la danse et de la musique ont des longueurs différentes. Par exemple, une phrase de la danse peut occuper $7,71 / 2$, 9 ou ${ }^{9}$ mesures, tandis que la phrase musicale comprend constamment 4 ou 8 mesures. Ce décalage dimensionnel, qui peut survenir à des niveaux hiérarchiques divers, manifestant l'autonomie structurelle de la danse, est savouré avec plaisir par les danseurs, qui donnent l'impression de ne pas du tout écouter la musique.

Pendant la performance, les danses ne commencent jamais en même temps que la musique car le danseur a besoin d'un court instant pour sentir la musique et en saisir le rythme. Ce décalage de temps entre les unités structurelles de la danse et de la musique est appelé une « incongruité périodique » (Giurchescu \& Bloland $1995: 147-151)^{10}$.

La relation entre les unités structurelles de la danse et de la musique peut aussi être comprise et comparée en fonction de l'organisation interne. Les unités de forme de la danse et de la musique peuvent avoir une succession fixe ou libre. Les unités de la danse peuvent se dérouler en succession fixe et celles de la musique en succession libre ou, inversement, les unités de la danse en succession libre et celles de la musique en succession fixe ${ }^{11}$. Cet aspect du rapport entre la danse et la musique a une certaine importance, si l'on pense à la fonction régulatrice exercée par l'organisation interne de la 
musique d'accompagnement sur le déroulement de l'improvisation ou de la composition de la danse.

21 Le rôle des mélodies dans la relation entre la danse et la musique peut uniquement être analysé dans le cadre d'une culture donnée et dans une perspective diachronique. D'habitude, la mélodie sert à signaler un certain type de danse à une communauté donnée. C'est pourquoi les bons musiciens aident à garder vivante la tradition de la danse et peuvent réveiller dans la «mémoire kinesthésique » des danseurs des danses oubliées ou qui existent à l'état latent.

Fig. 3 : En dansant la fecioreasca (danse de jeunes gens) devant les musiciens (violon, contra, contrebasse), dans le village de Frata (Roumanie)

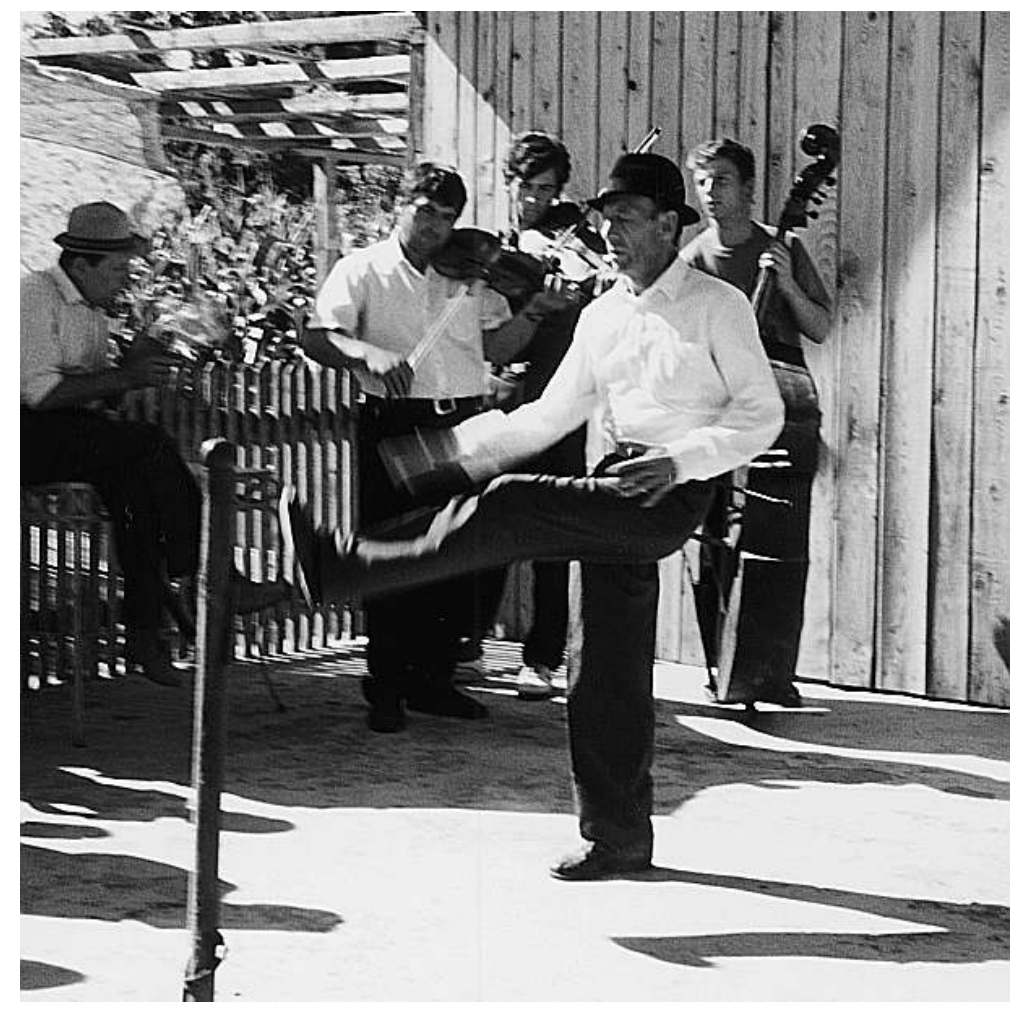

Photo : Anca Giurchescu

Lorsque la mélodie (vocale et instrumentale) et la danse font partie d'un «objet sémiotique » (spécialement dans les contextes rituels), elles ont la même fonction et sont donc intimement liées. Parmi les nombreux exemples d'une relation fixe entre la mélodie et la danse dans les contextes rituels, je ne citerai que les danses de guérison du rituel călu ş de Roumanie. Chaque danse, désignée par son nom, correspond à une mélodie qui signale le genre de mal à guérir et la source de la maladie (Giurchescu \& Bloland 1995 : 203). A l'inverse, la relation univoque entre la musique et la danse peut signifier un stade historique plus récent dans la culture occidentale. Dans d'autres cultures, elle caractérise une tradition ancienne comme celle de Tonga où le rapport fixe entre la mélodie, la poésie et les gestes est important pour transmettre le message de la danse et son esthétique (Kaeppler 1993 : 49).

23 Normalement la relation entre la mélodie et la danse est variable, dans le sens où une mélodie peut accompagner deux ou plusieurs différentes variantes (types) de la danse ou, au contraire, plusieurs mélodies différentes peuvent s'associer à un seul type de danse. 
Cette variabilité provient du fait que les types de danse imposent des contraintes essentiellement métriques et rythmiques à la musique (Felföldi 1995 : 193).

Il arrive fréquemment qu'un type de danse appelle différentes mélodies qui, après avoir transformé et adapté leur tempo, leur mètre, leurs dynamiques et leurs schémas rythmiques, sont adoptées par une communauté locale et reliées au type de danse correspondant. Etant donnée cette capacité transformatrice de la danse par rapport à un type mélodique, de nombreuses mélodies instrumentales pour la danse proviennent de chansons, de chants rituels ou de lamentations (Paska 1994 : 186). Nous ne citerons ici que quelques-uns des très nombreux types de danse possédant des centaines de variantes mélodiques : invârtita, hora, sârba (Roumanie), danses roumaines et hongroises de jeunes gens de Transylvanie, csárdás (Hongrie), Ländler (Allemagne) et Tarantella (Italie).

Fig. 4 : Cǎluşari et spectateurs dansant la hora autour de l'« étendard » et des musiciens, pendant le rituel de guérison căluş, dans le village d'Optaşi-Măgura (Roumaine).

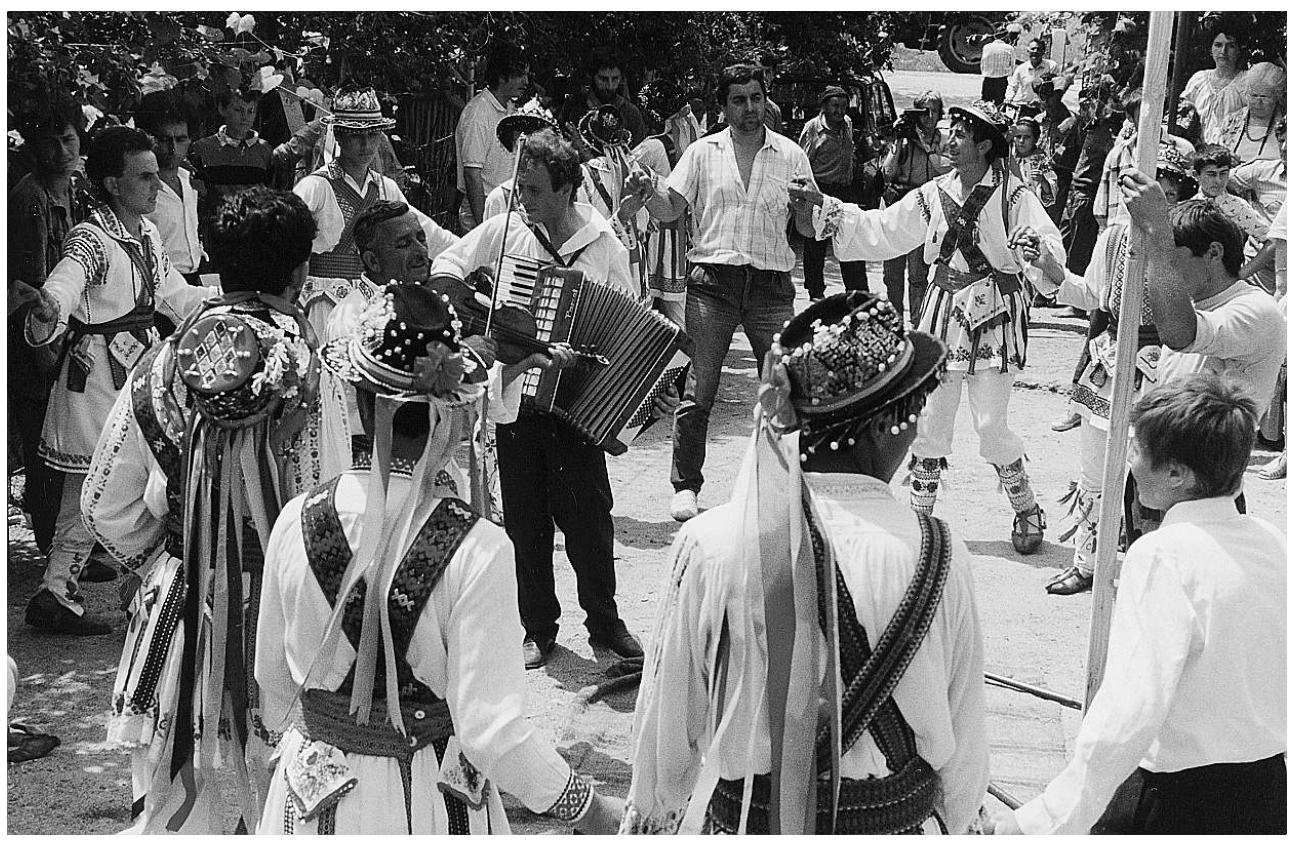

Photo: Helene Ericksen, juin 1993

A l'inverse, une seule mélodie (type mélodique) peut s'associer à plusieurs types de danses différents en subissant des modifications métriques et rythmiques. Néanmoins, l'essence mélodique qui est préservée peut parfois révéler des relations génétiques entre les types de danses. Par exemple, la danse processionnelle de couples de-a lungu et son dérivé, la danse tournante împiedecata de Transylvanie centrale, ont des mélodies communes ; ceci est également vrai pour la danse de jeunes gens (feciore şte) et la danse de couples purtata de Transylvanie méridionale qui ont les mêmes mélodies mais à des tempos différents.

La recherche au niveau de l'expression doit s'occuper de tous les aspects de la musique comme le rythme, la mélodie, l'harmonie, la dynamique, le tempo, le timbre particulier des instruments de musique, qui influencent le mode d'exécution et le contenu émotionnel d'une danse. Par exemple, le passage du chant à l'accompagnement instrumental au cours d'une même danse a un impact considérable sur la structure et le style de celle-ci (comme dans les danses en chaîne des femmes aux Balkans). L'expérience 
empirique permet de constater que le rythme asymétrique (c'est-à-dire $2+3 ; 1+1+1+2 ; 2+2$ $+2+3$ ) se traduit par le boitillement, par des mouvements irréguliers ou des mouvements ondoyants comme le schéma $2+2+3$ de la racenica bulgare. Un flux mélodieux et un tempo modéré favorisent une danse dont les mouvements sont soutenus et «élégants » comme dans la purtata roumaine et la polska suédoise. Les mouvements saccadés accompagnés de sauts et de torsions des différentes parties des membres et du corps de la danse hip hop correspondent à la musique rap et scratch (Giurchescu \& Torp 1995 : 148). L'augmentation du volume sonore et l'intensité de la musique d'accompagnement sont fréquemment suivies de mouvements extravertis et explosifs parfaitement illustrés par la danse des hommes bhangra du Punjab, en Inde, caractérisée par des mouvements librement improvisés, dirigés vers le haut, à l'adresse des dieux. Il faut également mentionner les nombreuses et différentes façons dont on utilise la musique pour provoquer divers types de transe et d'extase et entrer ainsi en communication avec les pouvoirs surnaturels ou les démons de l'au-delà (Rouget 1990 : 322-325).

\section{L'improvisation}

Il y déjà longtemps, l'ethnomusicologue allemand Felix Hœrburger exprima des doutes quant à l'existence d'une relation stricte entre la danse et la musique lorsque la danse est exécutée dans son contexte naturel. Tout en admettant qu' " un rythme rigoureux et une forme concise jouent un rôle important dans la musique de danse ", il soutient que ce rôle n'est pas déterminant et se demande si le danseur ne trouve pas plus de plaisir et d'inspiration dans des mouvements « aux rythmes libres » et dans une forme improvisée lui permettant d'exprimer son individualité et sa personnalité artistique. Il conclut: «Lorsque la danse et la musique de danse continuent à vivre librement de leur 'première existence', elles ne deviennent jamais des créations fixes et rigides, mais sont à chaque fois recréées par le détenteur de la tradition » (Hœrburger 1986 : 83, 96-97) ${ }^{12}$.

C'est au cours de l'improvisation que se révèle le mieux l'interaction entre un danseur et un musicien occupés à créer une nouvelle danse. Mais il est vrai aussi que l'improvisation n'est pas présente dans toutes les cultures, et que de nombreux genres de danse l'excluent. Si toutefois elle existe, ce type de relation est très subtil et complexe. Envisagé dans le cadre d'une culture traditionnelle, le concept d'improvisation peut se définir comme une activité créative consciente et intentionnelle qui intervient de façon spontanée et sans aucune préparation préalable pendant le déroulement de la danse, en utilisant des motifs de danse ou de plus grandes unités structurelles préexistantes pour composer de nouvelles séquences de mouvement ${ }^{13}$. Cela ressemble à un jeu: même spontanéité, même satisfaction de trouver des solutions personnelles en se servant du matériel (de danse) disponible et de règles grammaticales. La répétition exacte des séquences improvisées n'a que rarement lieu car la "fixation » ne veut rien dire pour le danseur qui improvise. En « jouant » avec et contre les caractéristiques structurelles de la musique, avec et contre les musiciens, les autres danseurs et les spectateurs, l'improvisateur tente de se démarquer des autres et d'exprimer sa personnalité.

L'improvisation a une double détermination: l'une située au niveau de la "parole », se rapporte à la compétence du danseur, à ses capacités d'exécution et à ses particularités psychosomatiques, l'autre, située au niveau de la «langue », se réfère au vocabulaire du mouvement et aux règles de la composition qui sont fixés par la tradition et qui facilitent la communication dans le cadre d'une culture de danse donnée. De ce point de vue, le 
rapport avec la musique sert d'une part à stimuler l'individualisation, d'autre part à instaurer de l'ordre et des règles à l'intérieur de l'improvisation. Dans les danses improvisées, la relation entre la musique et la danse est, par essence, un dérivé de l'interaction entre le musicien et le danseur, qui s'appuie sur un système de communication par signes et par codes. Elle est basée sur des valeurs esthétiques communes, des normes culturelles identiques, une expérience partagée et une longue pratique.

C'est une tâche essentielle du chercheur que de mettre en évidence la conceptualisation émique d'activités définies, en gros, comme étant de la «danse » et de la "musique de danse ", et de révéler leur processus de production dans différentes sociétés et en divers environnements sociaux. C'est donc sur le sens que les danseurs donnent eux-mêmes aux expressions verbales que s'appuient les remarques suivantes sur l'improvisation ${ }^{14}$.

31 Seuls des musiciens et des danseurs ayant de très grandes compétences dans leurs domaines respectifs peuvent collaborer pendant l'improvisation, car « un bon danseur et un mauvais musicien ne pourraient jamais coexister.» Pour être acceptés par la communauté, les musiciens doivent connaître les airs, le rythme, le tempo et la dynamique propres à chaque type de danse. Ils sont souvent de très bons danseurs et, même si ce n'est pas le cas, ils connaissent parfaitement la danse : «Vous ne pouvez pas jouer de la musique pour la danse, si vous ne savez pas danser vous-mêmes. » Ceci est également vrai pour les danseurs. Ils doivent avoir le sens du rythme " pour toucher le sol exactement quand l'archet frappe la corde » et le sens de la structure mélodique : «Vous ne pouvez changer que lorsque la mélodie vous dit de le faire.» En fait, danseur et musicien sont censés créer en parfaite harmonie, chacun menant l'autre et le suivant à tour de rôle, ce qui aboutit à un spectacle perçu par les spectateurs comme un tout auquel participent conjointement la musique et tous les autres moyens d'expression: «La musique, les oreilles et les jambes forment ensemble un tout !»

Je tenterai d'expliquer certains aspects de la collaboration entre le danseur et le musicien. En ce qui concerne la proxémique, les meilleurs danseurs se battent quasiment pour danser devant le musicien, dans un " espace d'honneur " symbolique où le danseur entre dans la sphère de la musique et noue un contact subtil avec le musicien principal. Ils jouent et dansent l'un pour l'autre, joutent l'un avec l'autre et présentent ensemble un spectacle à l'assistance. 
Fig. 5 : Le danseur Vasile Soporan (cf. note 15) accompagne du claqement rythmique de ses doigts les mouvements improvisés de la danse de jeunes gens, lors d'une réunion informelle.

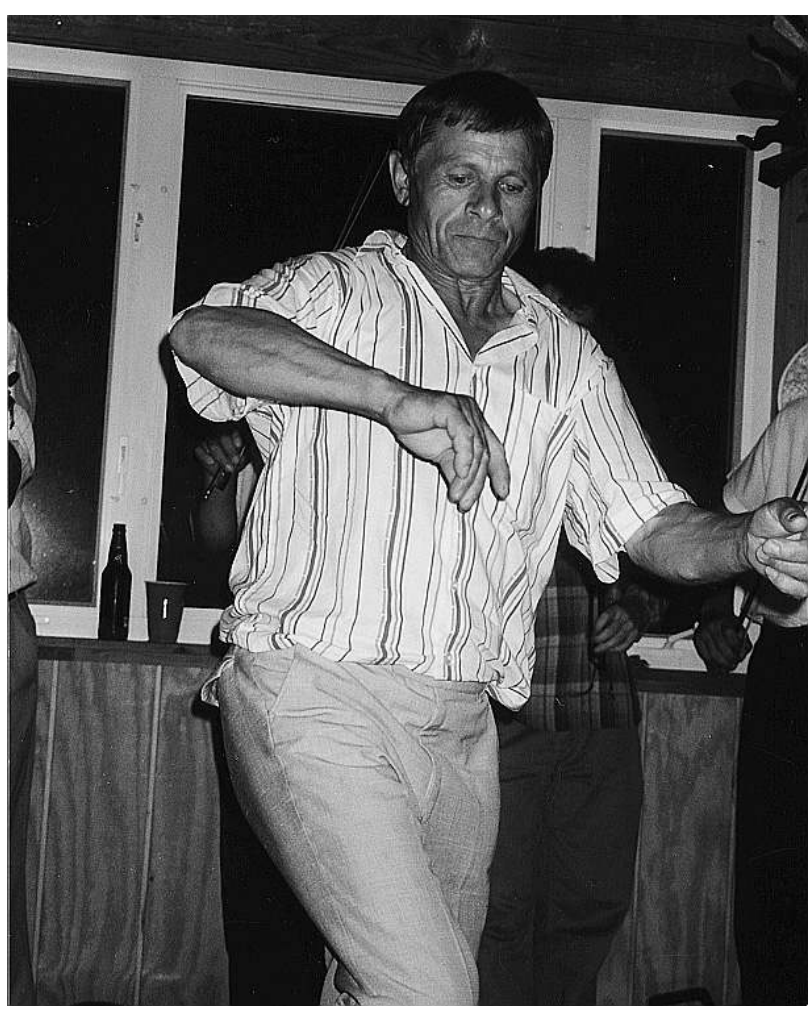

Photo: Sunni Bloland, juin 1999

Dans certaines traditions comme celles des danses polonaises et slovaques, le musicien est subordonné au danseur. Celui-ci commence par chanter sa mélodie, que reprennent ensuite les musiciens pour accompagner la danse. Il indique aussi le tempo et la dynamique qui correspondent à son humeur, à sa psychologie et à sa capacité physique. Le danseur peut accélérer ou ralentir le tempo, "étirer» ou "rétrécir» le schéma rythmique et métrique dans une sorte de rubato; il peut aussi faire des pas à contretemps. Ces «licences poétiques» sont suivies de près par des musiciens compétents (Lange 1975 : 35). Le musicien doit surveiller le danseur et adapter le tempo : il existe en effet une vitesse optimale qui permet au danseur de "boucler» tous les mouvements. Pour inciter un danseur à improviser, le musicien joue sa mélodie favorite : «Il joue pour moi avec passion tous les airs qu'il connait. Je me sens comme transporté d'une mélodie à l'autre. Il me signale à moi seul qu'il va maintenant jouer 'ma' mélodie, celle que j'aime le plus. » C'est ainsi que l'on trouve plus d'une mélodie baptisée du nom d'un bon danseur.

Dans l'optique du musicien, c'est lui qui domine le danseur : «Je dirige, je guide un bon danseur. Je lui donne force et inspiration. Seulement si le danseur n'est pas assez bon je l'aide en ralentissant le tempo et en en jouant plus simplement et de façon plus directe ", dit le violoniste Alexandru Ciurcui-şandorică de Soporul de Câmpie, district de Cluj (1995). Afin d'établir une communication expressive avec le danseur, le musicien doit deviner ses intentions en " lisant » le langage de son corps, en écoutant tous ses signaux sonores et tous ses ordres verbaux pour faire les ajustements nécessaires. De plus, en suivant le danseur, le musicien ressent lui-même une sensation kinesthésique d'énergie et 
de rythme qu'il exprime par les mouvements subtils de son corps et en battant le rythme de la danse avec les pieds : " Je suis inspiré quand je joue pour un bon danseur. »

$\mathrm{Au}$ cours de l'improvisation, le rythme présente la surface de contact la plus importante entre les danseurs et les musiciens qui s'appuient sur lui et jouent avec lui. Les modalités les plus courantes de l'improvisation rythmique sont la division de la durée fondamentale, l'accentuation à contre-temps et la syncope. Lorsque le rythme de la musique est accentué par les instruments d'accompagnement, le danseur accentue à son tour le rythme de son corps en tapant du pied, claquant les talons, battant des mains, claquant les doigts et en frappant ses jambes et les autres parties de son corps avec les mains. A ces rythmes audibles s'en ajoutent d'autres, produits par les mouvements insonores des bras, des mains, des hanches etc., et qui nous représentent le danseur tel un orchestre complexe jouant une partition à portées multiples. Pour autant que des paroles spontanées et des cris versifiés viennent s'ajouter à la danse, la superposition de leurs schémas rythmiques à ceux de la musique et du corps «orchestral» crée un effet polyrythmique et polymétrique fascinant et passionnant.

Dans la danse et la musique, l'improvisation rythmique est alternative plutôt que simultanée. Le schéma rythmique ostinato répétitif de l'accompagnement musical offre au danseur un support et l'incite à improviser. Les spectateurs apprécient cette interaction et la qualifient de «bonne improvisation». Le support rythmique est tellement important pour le danseur improvisateur que souvent, il prête à peine attention à la mélodie. Le danseur (aussi bien que le musicien) «pense » en motifs et s'en sert comme des unités constructives à part entière, les répétant et les transformant à loisir. Mais il ne faut pas croire qu'en danse l'improvisation soit un flux continu de rythmes et de mouvements nouveaux et inattendus. Pour construire mentalement de nouvelles images de danse, l'improvisateur a besoin d'un temps de repos basé sur la reprise et la répétition de motifs redondants, lui permettant d'étayer une idée artistique et de prendre un peu de recul pour planifier à l'avance. C'est pourquoi la forme de danse la plus fréquente créée lors d'une improvisation est le rondo. La structure mélodique de la musique, ses demi-cadences et ses cadences servent à modeler les unités les plus grandes de la forme de danse improvisée (telles que les phrases et les sections). Soulignant le rôle inspirant et régulateur de la mélodie, Soporan déclare : « Maintenant, à mon âge avancé, j'ai inventé des 'figures' que je n'avais jamais réalisées auparavant, je les ai créées parce qu'elles correspondaient parfaitement à la musique... » En guise de conclusion, j'aimerais citer une autre phrase du danseur sur son rapport avec le musicien pendant la danse : «Le violoniste établit le contact avec le danseur bien qu'ils ne se regardent pas dans les yeux. Il écoute le bruit des pas et les accentue avec son archet. Il me fait mieux danser, avec plus de conviction. Nous nous stimulons mutuellement. Si le violoniste est bon et que tu es entièrement pris par la danse, toute ta science et ton imagination sont mises en œuvre... Si tu danses bien, le violoniste joue avec plus d'entrain pour toi que pour les gens qui sautent devant lui comme du pop-corn sur le feu.» 


\section{BIBLIOGRAPHIE}

CIOBANU Gheorghe, 1979, « Inrudirea dintre ritmul dansurilor şi al colindelor » (The relationship between the rhythm of dances and carols). Studii de etnomuzicologie şi bizantinologie, (Ethnomusicology and Bysantinology Studies) vol. II. Bucure şti: Editura Muzicală: 64-94.

DABROWSKA Grazyna \& Kurt PETERMANN eds, 1983, « Grundlagen der Struktur- und Formanalyse des Volkstanzes ». Analyse und Klassifikation von Volkstänzen. Kraków: PWM: 28-31.

FELFÖLDI László, 1995, « The Connection of Dance and Dance Music Over or Under-Estimation of their Significance ", in Grazyna Dabrowska, Ludwik Bielawski eds : Dance, Ritual and Music. Proceedings of the 18th Symposium of the ICTM Study Group on Ethnochoreology (Skierniewice, Poland, 1994). Warszawa: Instytut Sztuki PAN: 189-195.

GIURCHESCU Anca, 1967, « Purtatele de fete de pe Valea Tîrnavelor » (Girl's Purtate of Tîrnava Valley), Revista de Etnografie şi Folclor (Bucure şti), 12 (4): 283-297.

GIURCHESCU Anca, 2000, «Interpreting a Dancer's Discourse on Improvisation », Dans Müzik Kültür. ICTM 20th Ethnochoreology Symposium Proceedings 1998, Frank Hall and Irene Loutzaki eds: 259-274. Istanbul: Bogaziçi University Folklore Club., GIURCHESCU Anca and Lisbet TORP GIURCHESCU Anca, 1995, « Dance-Music Relationships: An Introduction », in Grazyna Dabrowska, Ludwik Bielawski eds : Dance, Ritual and Music. Proceedings of the 18th Symposium of the ICTM Study Group on Ethnochoreology (Skierniewice, Poland, 1994). Warszawa : Instytut Sztuki PAN :143-149.

GIURCHESCU Anca and Sunni BLOLAND, 1995, Romanian Traditional Dance. A Contextual and Structural Approach. Mill Valley, CA : Wild Flowers Press.

GREIMAS A.-J., 1971, « Réflexions sur les objets ethno-sémiotiques (Manifestations poétique, musicale et gestuelle)». Actes du premier Congrès International d'Ethnologie Européenne (Paris) : 63-72.

HOERBURGER Felix, 1986, "Gestalt und Gestaltung im Volkstanz », in Hans Eichner and Thomas Emmerig eds : Volksmusikforschung. Aufsätze und Vorträge 1953-1984 über Volkstanz und instrumentale Volksmusik. Laaber : Laaber-Verlag : 83-88 [First published in Studia musicologica 6, 1964: 311-316].

KAEPPLER Adrienne L., 1993 , Poetry in Motion : Studies of Tongan Dance. Tonga: Vava'u Press.

KEALIINOHOMOKU Joann, 1965-66, « Dance and Self-Accompaniment ». Ethnomusicology IX-X: 292-295.

KRÖSCHLOVA Eva, 1983, « Diskussionbeitrag zu den Grundlagen der Struktur- und Formanalyse des Volkstanzes ", in Grazyna Dabrowska und Kurt Petermann eds : Analyse und Klassifikation von Volkstänzen. Kraków: PWM: 50-55.

KURATH Gertrude Prokosch, 1986 , « Dance Relatives of Mid-Europe and Middle America: A Venture in Comparative Choreology ». Half a Century of Dance Research. Arizona: Cross Cultural Dance Resources: 308-320.

LANGE Roderyk, 1975, The Nature of Dance. An Anthropological Perspective. London: Macdonald \& Evans.

MARTIN György, 1965, « Considérations sur l'analyse des relations entre la danse et la musique de danse populaires ». Studia Musicologica VII (Budapest) : 315-338. 
MARTIN György, 1968, « Die ungarischeMädchenreigen » Veröffentlichungen des Instituts für Volkskunde der Universität Wien, Band 2 : 325-343.

MARTIN György, 1973, « Die Branles von Arbeau und die ost-europäischen Kettentänze ». Studia Musicologica XV VII (Budapest): 101-128.

MICHAELIDES Solon, 1978, The Music of Ancient Greece : an encyclopaidia. London: Faber.

MÎRZA Traian, 1972, « Ritmul orchestic (de dans); un sistem distinct al ritmicii populare române şti » (The rhythm orchestic (dance) ; a distinct systhem of the Romanian folk music). Studii de muzicologie, (Musicology Studies). Bucure şti, Editura Muzicală 8: 231-262.

NILSSON Mats, 1995, « Let's Meet in the Beat », in Grazyna Dabrowska, Ludwik Bielawski eds : Dance, Ritual and Music. Proceedings of the 18th Symposium of the ICTM Study Group on Ethnochoreology (Skierniewice, Poland, 1994). Warszawa: Instytut Sztuki PAN: 211-216.

PASKA Katalin, 1994, « How folk dance transforms its related music ». Acta Ethnographica Hungarica 39 (1-2) : 185-204.

ROUGET Gilbert, 1990, La musique et la transe : esquisse d'une théorie générale des relations de la musique et de la possession. Paris: Gallimard. (Nouv. éd. rev. Et augm)

SACHS Curt, 1952, World History of Dance. New York: Seven Arts Publishers.

STOCKMANN Doris, 1985, « Music and Dance Behaviour in Anthropogenesis ». Yearbook for Traditional Music 17:16-30.

TABOUROT Jean (Thoinot Arbeau), 1970, Orchésographie; réimpression. Genève : Slatkine Reprint. (Reprod. Photomécanique de l'éd. de Paris 1888. L'éd. originale est de Langres, 1589).

TORP Lisbet, 1990, Chain and Round Dance Patterns. A Method for Structural Analysis and its Application to European Material. vol. I-III. University of Copenhagen : Museum Tusculanum Press.

\section{NOTES}

1. Il faudrait aussi mentionner le terme orchestics en grec ancien, défini par Curt Sachs (1953 : 27-28) comme « un agrégat organique de poésie, de musique et de danse qui, en se combinant, révèle l'unité corporelle et spirituelle de l'homme».

2. En s'appuyant sur le syncrétisme de la danse, de la musique et de la poésie encore présent dans la tradition de la danse roumaine, l'ethnomusicologue Trajan Mîrza a démontré l'existence d'un système rythmique distinct, appelé « rythme de danse » (orchestique), qui est fondamentalement différent $\mathrm{du}$ système métrique occidental. Alors que le système rythmique occidental est divisionnaire, avec une distribution périodique des accents, le "rythme de danse ", lui, est «bichrone", avec les noires et les croches comme valeurs de base dans un rapport de $1: 2$ et $2: 1$ et la formule $8 / 8(6 / 8)$ comme cadre rythmique et métrique le plus constant. Il est fondé sur le principe additif et non divisionnaire (proche du système indien) et fait preuve d'une grande variété dans la distribution des accents (Mîrza 1972).

3. La danse "silencieuse » fut exécutée par les Monténégrins au Danemark, le 18 avril 1987, pendant l'événement chorégraphique des Vlachs, appelé hora. (Les Vlachs sont une population de langue roumaine du Nord-Est de la Serbie, établie comme travailleurs immigrés au Danemark depuis 1964). L'énergie contenue et pourtant intense de la danse "silencieuse " laissa non seulement une forte impression esthétique sur les spectateurs, elle fut encore significative en tant que symbole de l'identité et des différences entre hommes et femmes. 
4. Lorsque la relation entre la danse et la musique de danse est étudiée in vitro (d'après les enregistrements), il est crucial, afin d'éviter toute distorsion, d'enregistrer la musique pendant le spectacle de danse car la même mélodie est jouée différemment selon qu'elle est destinée à la danse ou à l'écoute. Dans ce dernier cas, elle est souvent jouée plus librement et en respectant moins strictement le tempo.

5. L'effet impressionnant des mouvements rythmiques accentués par des sons a développé, spécialement pendant le déroulement de la danse sur scène, ce qu'on appelle une série " break » de mouvements de battements et de claquements, qui frappe beaucoup les spectateurs.

6. En 1971, assistant par un jour de marché à une danse accompagnée par un flûtiste, dans le village de Vaideeni du district de Gorj, en Roumanie, j'eus l'occasion de constater que, pendant la pause du musicien qui dura une trentaine de minutes, les danseurs continuaient à exécuter la danse tournoyante invârtita en s'accompagnant uniquement du rythme des cris versifiés qu'ils poussaient eux-mêmes.

7. Il n'est pas sans intérêt de constater que la plupart des musiciens qui accompagnent la danse battent du pied le schéma rythmique de celle-ci et non le rythme de la musique d'accompagnement.

8. Un des critères selon lesquels les danses sont organisées en suites et en cycles fixes est l'interaction dynamique, c'est-à-dire l'arrangement des danses en fonction du contraste, de l'alternance ou de l'augmentation progressive du tempo et de l'intensité du mouvement (Giurchescu 1995 : 145).

9. L'ethnomusicologue roumain Gheorghe Ciobanu a avancé l'hypothèse que la structure hexasyllabique des chants de Noël pourrait suggérer qu'à l'origine, ils étaient accompagnés de danses.

10. Par exemple, dans la tradition roumaine, la danse commence d'habitude sur la deuxième ou la quatrième mesure de la première (parfois de la deuxième) ligne mélodique avec un motif correspondant. La ligne musicale suivante et la figure de la danse évoluent ensuite de pair.

11. La relation entre les formes de la danse et de la musique en fonction de la dimension, de la succession et de l'organisation interne de leurs unités structurelles a été analysée par le groupe d'étude de l'ICTM sur l'ethnochoréologie et publiée dans Analyse und Klassifikation von Volkstänzen (1983 : 28-31), auquel Eva Kröschlova ajouta dans le même volume «Discussionbeitrag zu den Grundlagen der Struktur- und Formanalyse des Volkstanzes » (ibid. :50-55).

12. S'appuyant sur l'étude de la danse "populaire» dans la ville de Göteborg, en Suède, l'ethnologue Mats Nilsson tire la conclusion suivante: "Dans la danse populaire vivante et toujours pratiquée, les danses sont reliées à la musique essentiellement par la pulsation, alors que dans la danse apprise à l'école, les danses correspondent plus à d'autres parties de la musique, telles que la mesure, le rythme, la phrase, etc. Il fait aussi cette remarque : «La danse folklorique et la musique sont très intégrées; oui, elles le sont, mais peut-être seulement 'dans la tête' et non 'sur le sol'" (Nilsson 1995 : 212-213).

13. Adrienne Kaeppler définit l'improvisation comme «la chorégraphie spontanée de morceaux de danse acceptables culturellement pour des genres spécifiques » (Kaeppler 1987 : 21).

14. Les citations utilisées dans cet article sont extraites d'interviews sur l'improvisation réalisés en 1995 avec le danseur Vasile Soporan, né en 1932, dans la commune de Frata du district de Cluj (Giurchescu $2000: 269$ ). 


\section{RÉSUMÉS}

$\mathrm{Au}$ niveau de la communication folklorique, des activités artistiques pouvant fonctionner de façon autonome dans d'autres contextes sociaux agissent les unes par rapport aux autres comme parties intégrantes d'un "texte culturel» unitaire générant une signification globale. L'importance des modalités expressives, dont font partie la danse et la musique, varie d'une culture à l'autre, montrant par là que l'art est moins universel qu'on le prétend. L'unité entre la danse et la musique (pertinente pour la culture européenne) ainsi que la relation danseur/ musicien sont étudiées ici en situation d'interprétation, spécialement au cours de l'improvisation.

Le point de rencontre le plus important entre danse et musique, danseur et musicien est le rythme, qui crée des effets polyrythmiques et polymétriques, surtout pendant les improvisations. La structure et la forme sont ici comparées en fonction de la coïncidence ou de l'absence de coïncidence dimensionnelles entre les unités constitutives de la danse, de la musique et des textes. La relation variable entre les mélodies et les danses découle du fait qu'une mélodie peut accompagner différents types de danse et que plusieurs types mélodiques peuvent s'associer à un seul type de danse.

La dernière partie de l'article est consacrée au processus de l'improvisation et à la relation entre le danseur et le musicien, qui est fondée sur une série de normes culturelles et sociales communes, sur des affinités esthétiques, sur la compétence et une longue pratique.

\section{AUTEURS}

\section{ANCA GIURCHESCU}

Anca GIURCHESCU, ethnochoréologue, est née en Roumanie où, directrice de recherches à l'Institut d'Ethnographie et du Folklore à Bucarest, elle contribua de 1953 à 1979 à l'élaboration de la recherche scientifique sur la danse traditionnelle en Roumanie. Installée au Danemark depuis 1980, elle continue d'étudier les Vlachs, une minorité ethnique de langue roumaine venue du Nord-Est de la Serbie et résidant actuellement au Danemark, tout en faisant des recherches sur les Tziganes de Roumanie. Elle a publié de nombreux articles sur la danse et le rituel (notamment le căluş), des analyses structurelles de la danse ou encore sur la danse et l'identité et l'utilisation de la danse à des fins de légitimation du pouvoir politique. Elle préside le Groupe d'étude sur l'Ethnochoréologie du Conseil International de la musique traditionnelle et donne de nombreuses conférences à l'échelle internationale. 